\title{
Coupling of CSF and sagittal sinus pressure in adult patients with pseudotumour cerebri
}

\author{
Afroditi-Despina Lalou ${ }^{1,2,3,4}$ - Marek Czosnyka ${ }^{1,2,3} \cdot$ Zofia H. Czosnyka $^{1,2,3}$ - Deepa Krishnakumar ${ }^{1,2,3}$. \\ John D. Pickard ${ }^{1,2,3} \cdot$ Nick J. Higgins ${ }^{1,2,3}$
}

Received: 15 May 2019 / Accepted: 8 October 2019 /Published online: 12 December 2019

(C) The Author(s) 2019

\begin{abstract}
Objective Pseudotumour cerebri syndrome (PTCS including idiopathic intracranial hypertension) is characterised by the symptoms and signs of raised cerebrospinal fluid pressure (CSFp) in the absence of ventricular dilatation or an intracranial mass lesion. Its aetiology is unknown in the majority of cases but there is much evidence for impaired CSF absorption. Traditionally, sagittal sinus pressure has been considered to be independent of CSF pressure in adults. However, the discovery of stenoses of intracranial venous sinuses and introduction of venous sinus stenting has highlighted the importance of the venous drainage in PTCS. In this study, we have explored the relationship between CSFp and SSp before and during a CSF infusion test and during CSF drainage.

Materials and methods Ten patients (9 females: 1 male) with PTCS underwent infusion studies in parallel with direct retrograde cerebral venography. Both SSp and CSFp were recorded at a baseline and during CSFp elevation in a course of a CSF infusion test. The drainage of CSF after the CSF infusion was performed in 7 patients. In 5 cases, jugular venous pressure was also measured.

Results CSFp and SSp including their amplitudes correlated significantly and strongly both at baseline $(R=0.96 ; p=0.001)$ and during infusion $(R=0.92 ; p=0.0026)$. During drainage, this correlation was maintained until SSp reached a stable value, whereas CSFp continued to decrease.

Conclusions In this series of ten patients with PTCS, CSFp and SSp were coupled, both at baseline and during infusion. The implications of such coupling for the calculation of CSF outflow resistance are discussed.
\end{abstract}

Keywords CSF pressure · Intracranial pressure · Idiopathic intracranial hypertension · Pseudotumour cerebri · Sagittal sinus pressure $\cdot$ CSF outflow resistance

Publication history: A preliminary report of our findings was presented at Intracranial pressure and Brain Monitoring XIII (Acta Neurochir Suppl. 2008; 102:283-5 PMID:19388330) and presented at Hydrocephalus 2018 in Bologna, Italy.

This article is part of the Topical Collection on CSF Circulation

Afroditi-Despina Lalou

ad143@cam.ac.uk

1 Division of Neurosurgery, Department of Clinical Neurosciences, Cambridge University Hospital, Cambridge, UK

2 Department of Paediatric Neurology, Cambridge University Hospital, Cambridge, UK

3 Department of Radiology, Addenbrooke's Hospital, Cambridge, UK

4 Newnham College, University of Cambridge, Sidgwick Avenue, Cambridge, Cambridgeshire CB39DF, UK

\section{Introduction}

Pseudotumour cerebri syndrome (PTCS) is characterised by the symptoms and signs of raised cerebrospinal fluid pressure (CSFp) in the absence of ventricular dilatation or an intracranial mass lesion and often without a known aetiology [10, 15, $16,27,28]$. It mostly affects women of reproductive age with an increased body mass index (idiopathic intracranial hypertension, IIH) but less frequently can involve paediatric patients and patients regardless of their biological sex [15-17]. MRI reveals dilatation of the cortical subarachnoid space and optic nerve sheaths with compression of the pituitary gland (empty sella) [9].

Potential underlying mechanisms for PTCS include impaired CSF drainage and raised intracranial venous sinus pressure $[12,13,25,26]$. There is little evidence for CSF 
hypersecretion or cerebral oedema. There is debate as to whether changes in CSF outflow resistance are sufficient per se to explain the raised CSF pressure in all patients [23]. Importantly, impaired CSF absorption may be caused by raised intracranial venous sinus pressure. Early studies of PTCS demonstrated abnormalities of intracranial venous drainage but later MR venography studies were misinterpreted as flow artefacts $[1,11,17,24,31]$. However, retrograde cerebral venography and CT venography demonstrated stenoses of the transverse sinuses in many patients with PTCS [12, 13, 25]. Commonly, patients with PTCS that is refractory to medical management have been offered surgery including various CSF diversion procedures and bariatric surgery $[10,31]$. The demonstration of venous sinus stenoses with significant pressure gradients led to the introduction of venous sinus stenting in 2002 [13, 18].

Many of these stenoses were not fixed but resolved, at least in part, with drainage of CSF. Such reversible stenoses contradict the traditional view that the intracranial venous sinuses are largely incompressible in adults with sagittal sinus pressure being independent of CSF pressure [24]. Furthermore, these stenoses might create a positive feedback loop between increased CSFp and SSp in patients with PTCS with secondary impairment of CSF absorption [28]. There is increasing debate in developing the evidence behind shunting, therefore decreasing CSFp, and stenting, therefore decreasing venous pressure and 'normalising' cerebral venous anatomy and compliance, in PTCS. On the other hand, there is very little evidence on the pathophysiology of the increase of the two pressures, how they interact, and why each of the selected treatments could be effective or not.

In this study, we have explored the degree of coupling between CSFp and SSp waveforms in adults suffering from PTCS by using lumbar CSF infusion studies to measure CSFp with simultaneous direct measurements of their $\mathrm{SSp}$ at baseline, during infusion, and during/after CSF drainage. In addition, we have examined the implications of such coupling for the calculation of CSF outflow resistance when using Davson's equation: CSFp $=$ Rout $\times I_{\mathrm{f}}+$ $\mathrm{SSp}$, where Rout is the resistance to CSF outflow and $I_{\mathrm{f}}$ is the CSF formation rate [6]. [28].

A preliminary account of this work has been published

\section{Patients and methods}

Between 2004 and 2006, we investigated 10 patients (9F:1M) with the clinical features of PTCS who fulfilled the modified Dandy criteria (signs and symptoms of raised ICP, no localising neurological signs, normal neuroimaging apart from MR venography, raised CSF pressure (> $20 \mathrm{mmHg}[8$, $10,15,, 27])$, and normal CSF constituents). Their mean age was 41 years (range 22-55). All the patients had both headaches and papilloedema.

As part of their clinical investigation, they underwent two procedures: constant-rate lumbar CSF infusion studies, to assess the CSFp and CSF dynamics, and direct retrograde cerebral venography (DRCV) whereby a catheter was placed within the sagittal sinus under fluoroscopic guidance, in order to assess the significance of the stenosis.

Patients undergo either or both procedures in our hospital routinely as part of their PTCS investigations, in order to establish a diagnosis and plan treatment. Both DRCV and lumbar infusions have been used safely in our centre as well as other centres internationally $[2,4,18]$.

\section{Lumbar infusion studies $[2,4,7]$}

Access was gained via lumbar puncture using two 21gauge Quincke needles at the intervertebral space L4L5 using lidocaine local anaesthesia, with the patient lying on their side. A strict aseptic technique was used to keep all the pre-filled tubing and the transducer sterile. The skin was carefully prepared with antiseptic solution. Connection of a standard, disposable fluid-filled pressure transducer (Edwards Lifesciences ${ }^{\mathrm{TM}}$ manometry lines, length $180 \mathrm{~cm}$ and inner diameter $1.2 \mathrm{~mm}$ ), and pressure amplifier (Spiegelberg or Philips) to the LP needle allowed for pressure recording at a frequency of 30 $100 \mathrm{~Hz}$, with following processing by ICM+ (University of Cambridge Enterprise Ltd.) [4, 30].

Once a satisfactory CSFp pulse waveform had been achieved, baseline measurements were taken for $10 \mathrm{~min}$, followed by infusion of Hartmann's solution at $1.5 \mathrm{ml} / \mathrm{min}$ or $1.0 \mathrm{ml} / \mathrm{min}$ if the baseline CSF was $\geq 15 \mathrm{mmHg}$ until the ICP had plateaued for 5-10 min. The protocol included a safety measure that required the infusion to stop if the mean ICP increased to $40 \mathrm{mmHg}$ or above. This did not occur in any of these patients. The total duration of the infusion tests was 30 to $45 \mathrm{~min}$. After the end of the infusion test, pressurecontrolled withdrawal of CSF was carried out without removing the pressure transducer, via a tap connected to the pressure lines. This allowed us to assess CSFp while continuing to measure and record SSp during and after the end of CSF removal. Withdrawal was stopped when the pressure reached $\sim 10 \mathrm{mmHg}$ or if the patient started complaining of headaches and/or blurred vision.

\section{Direct retrograde cerebral venography [12-14]}

The catheter inserted for pressure measurement with DRCV was longer and narrower than the manometry lines used for CSF pressure. 
SSp was monitored and recorded with ICM+ in the same way as CSFp. The mean pressure level, slow vasogenic waves (period from $20 \mathrm{~s}$ to $2 \mathrm{~min}$ ), and amplitude of pulse waveform $\left(\mathrm{AMP}_{\mathrm{SSp}}\right)$ were extracted through computer data analysis and recorded alongside CSFp.

\section{Statistical analysis}

Data points of all parameters were distributed normally and hence the paired Student $t$ test comparison was used for assessing the significance of any differences in pressures. A simple linear regression model was used to assess any associations between pairs of data.

\section{Ethics statement}

All tests were performed as part of routine clinical management. All patients consented to the use of their data recordings for research purposes. At the time of this study, such consent did not include permission for data sharing.

\section{Results}

Overall, we observed time-related coupling between mean CSFp and SSp (Fig. 1a), slow waves of CSFp and SSp (Fig. 1b), and pulse waveforms of CSFp and SSp. Pulse waveforms increased during an increase in both pressures provoked by infusion. Specifically, in cases when SSp amplitude was detectable (it was not possible to record any amplitude of SSp in 3 out of 10 sessions), both waveforms were adjacent in their diastolic phases and divergent during systole (Fig. 1c).

Mean SSp correlated very strongly with the CSFp at baseline: $R=0.96 ; p=0.0001 ; N=9$. Also at baseline, the pulse amplitudes of CSFp and SSp were well correlated (amplitude of SSp at baseline was recorded in 7 cases) (Fig. 2a).

During infusion, the two pressures increased concomitantly $(R=0.92 ; p<0.003 ; N=7)$ and the changes of both pressures correlated strongly $(R=0.97 ; p=0.0007 ; N=6)$. The slopes of the amplitude - pressure lines, calculated from a simple, linear regression model between CSFp and AMP of CSFp, and SSp and AMP of SSp - also correlated strongly during infusion $(R=0.97 ; p<0.005 ; N=7)$ (Fig. 2b).

\section{CSFp - SSp .... [mmHg]}
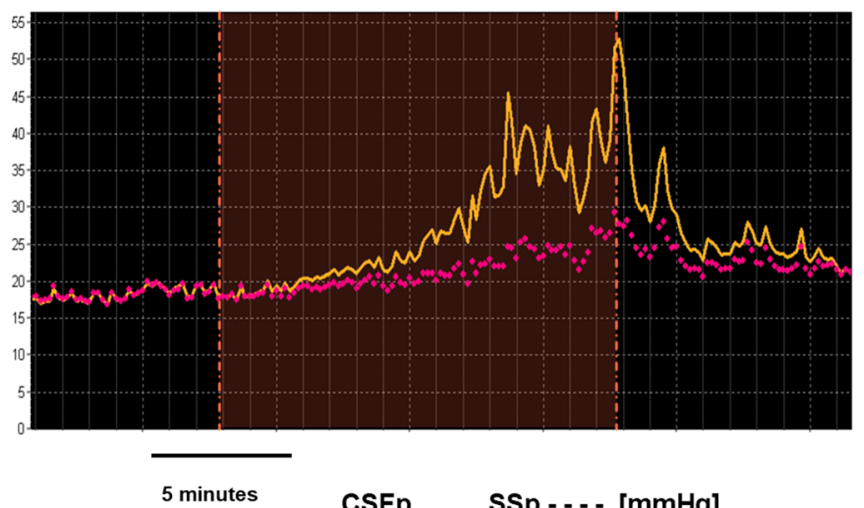

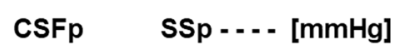

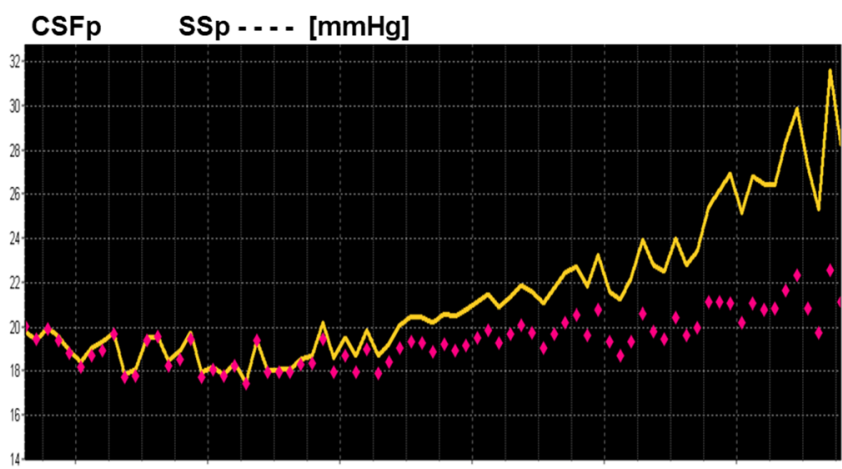

2 minutes

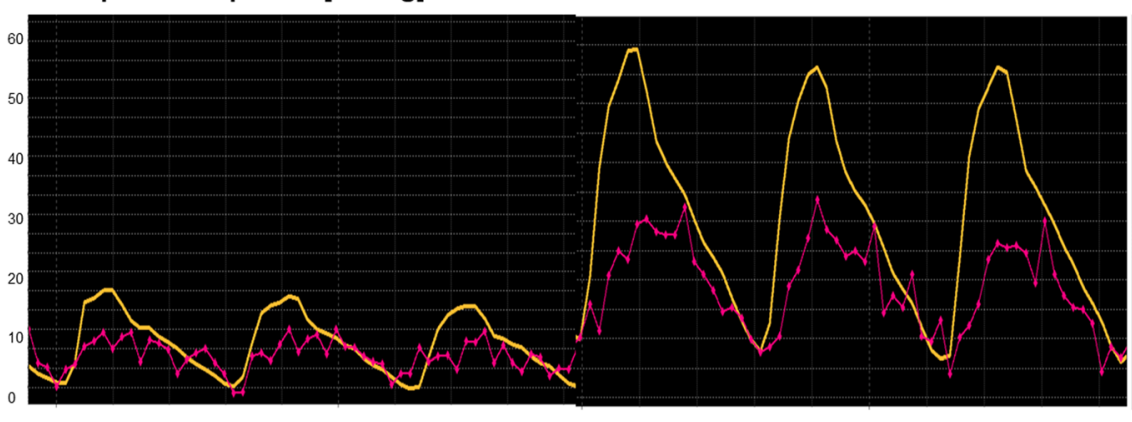

$1 \mathrm{sec}$

Fig. 1 Observational demonstration of the static and dynamic coupling between CSFp and SSp. a Static coupling between the mean CSFp (upper, darker trend) and mean SSp (lower, dotted trend) values at baseline, during and after the end of infusion (the infusion period is marked as an event represented by the white area in the graph). b
Dynamic coupling between the slow vasogenic waves of CSFp (upper, darker trend) and SSp (lower, dotted trend). c Coupling between the pulse amplitudes of CSFp and SSp at baseline and during infusion. CSFp, cerebrospinal fluid pressure; SSp, pressure of the sagittal sinus 
a

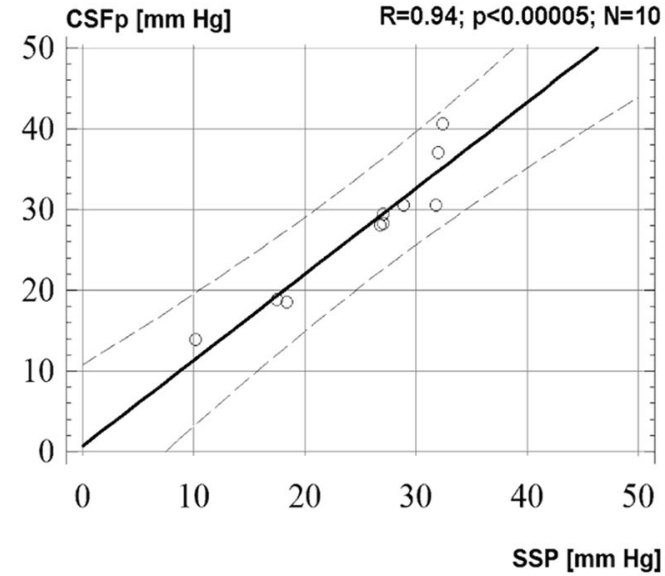

Pulse Amplitude of

CSFp [mm Hg]

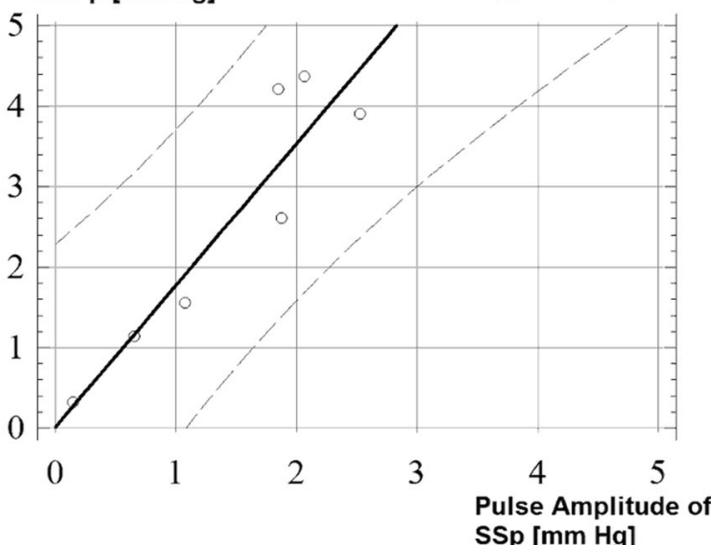

b

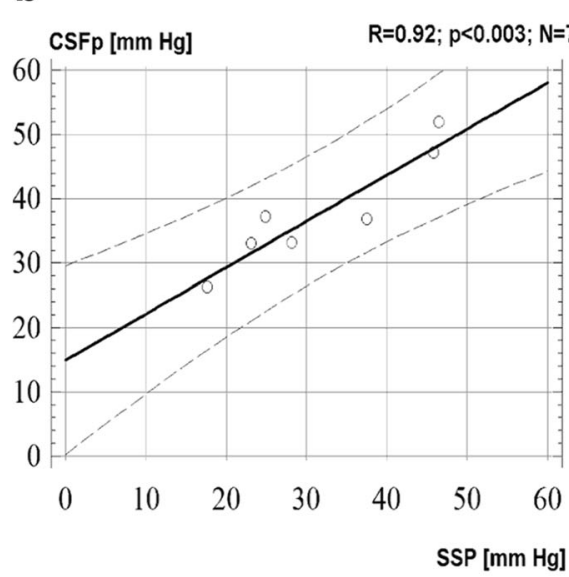

Fig. 2 Coupling of CSFp and SSp at baseline and during infusion. a Left: linear regression demonstrating the coupling between CSFp and SSp at baseline. Right: coupling between the pulse amplitude of CSFp and SSp at baseline. Recording of the amplitude was only possible in 7 out of the 10 cases. b Linear regression demonstrating the maintenance of the coupling between CSFp and SSp during infusion (left), the strong

Table 1 summarises the mean values and difference between CSFp and SSp at each phase of monitoring.

The jugular venous pressure was measured in 5 patients and on average was $10.43 \pm 3.8 \mathrm{mmHg}$. The jugular venous pressure (JVP) during one of the infusion tests is shown in Fig. $3 a$. Central venous pressure was measured on one patient and was relatively stable during infusion $(11.6 \pm 2.2 \mathrm{mmHg})$.
During drainage, the overall correlation between the 2 pressures was $R=0.78 ; p=0.065, N=6$ (Fig. 3b). During drainage, SSp appeared to stabilise at a level close to jugular vein pressure while CSFp continued to fall.

Table 2 summarises the differences between CSFp, SSp, and JVP at the end of drainage.

Table 1 Mean values of pressures during baseline, infusion and drainage

\begin{tabular}{llllll}
\hline & CSF pressure $(\mathrm{mmHg})$ & Sinus pressure $(\mathrm{mmHg})$ & $p$ value & CSFp-SSp $(\mathrm{mmHg})$ & $p$ value \\
\hline Baseline & $27.0 \pm 2.3$ & $25.2 \pm 7.5$ & $p=0.026 ; N=10$ & $2.34 \pm 2.72$ & 0.01953 \\
Infusion & $38.0 \pm 8.0$ & $33.1 \pm 12.0$ & $p=0.01 ; N=7^{*}$ & $4.9 \pm 4.0$ & $p=0.026 ; N=7 *$ \\
Drainage & $12.7 \pm 5.6$ & $16.0 \pm 2.7$ & $p=0.02 ; N=8$ & $-3.2 \pm 3.9$ & $p=0.0097 ; N=8$ \\
\hline
\end{tabular}

*In 3 patients, only drainage was performed, as baseline CSFp was $>40 \mathrm{mmHg}$

$C S F p$ cerebrospinal fluid pressure, $S S p$ pressure of the sagittal sinus 
a
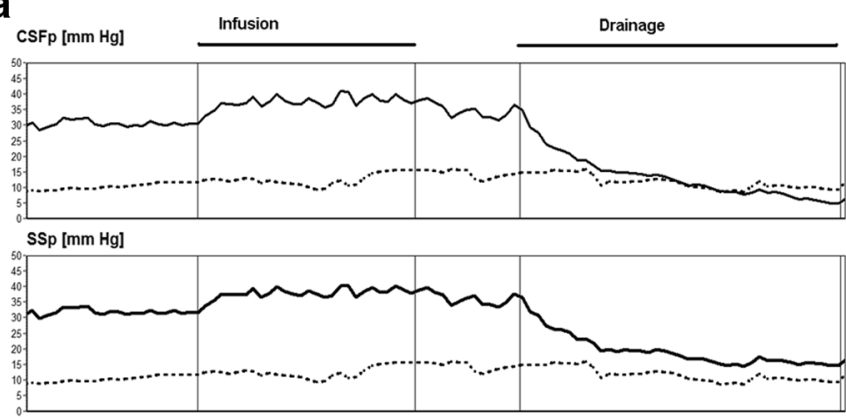

$\mathrm{JVp}[\mathrm{mm} \mathrm{Hg}]$

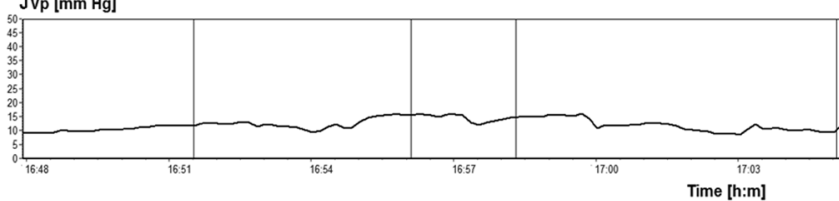

Fig. 3 Correlation between CSFp and SSp during CSF drainage. a Overview of CSFp and SSp and JVP during infusion and during drainage of CSF. JVP is projected as a dotted line on the CSFp and SSp (SSp) panels, demonstrating that CSFp continues to drop after reaching

\section{SSp as a function of CSFp}

Using linear regression for each individual patient, SSp was expressed as a function of CSFp in the format $\mathrm{SSp}=a \times \mathrm{CSFp}$ $+b$ (for example, Fig. 4).

In all patients who had undergone infusion and drainage, 'a' was calculated to be $0.70 \pm 0.14$ for 9 patients. 'b' was calculated as $6.3 \pm 3.5 \mathrm{mmHg}$ which represents the intercept of this correlation that physiologically should correspond to central venous pressure (CVP). In the one patient, CVP was measured and found to be $11 \mathrm{mmHg}$; the intercept of the correlation was $9.2 \mathrm{mmHg}$, which is within the limits of measurement error (e.g. zeroing of external transducers).

\section{Discussion}

Our results indicate the following, in many cases of PTCS:

- CSFp and SSp are coupled both statically (mean values) and dynamically (vasogenic components, mainly slow waves of CSFp and respiratory amplitude in CSFp and SSp).

- When CSFp increases during CSF infusion, it produces an increase in SSp and its vasogenic components.

Table 2 Differences between CSFp, SSp, and JVP at the end of drainage in $N=5$ patients with JVP measured

\begin{tabular}{lll}
\hline & $\begin{array}{l}\text { Difference } \\
(\mathrm{mmHg})\end{array}$ & $\begin{array}{l}\text { Significance } \\
\text { of difference }\end{array}$ \\
\hline CSFp-JVP (mmHg) & $-2.2 \pm 3.4$ & $p=0.026$ \\
SSp-JVP (mmHg) & $4.27 \pm 3.0$ & $p=0.004$ \\
\hline
\end{tabular}

b

CSFp during drainage $[\mathrm{mm} \mathrm{Hg}]$

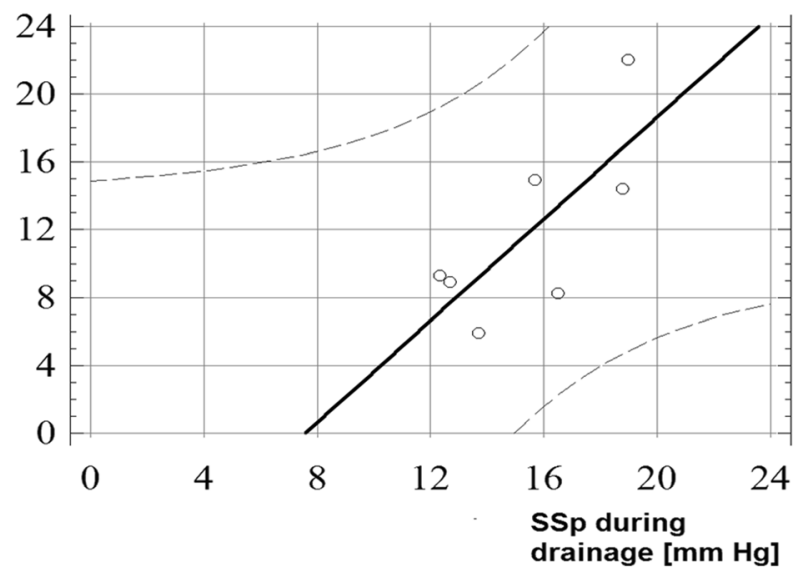

JVP; in contrast, SSp reaches values close to JVP (CVP) and remains stable at this value as CSFp continues to decrease. $\mathbf{b}$ Correlation between CSFp and SSp during drainage of CSF. JVP, jugular venous pressure

- During drainage, both pressures decrease until a certain point (most probably JVP) when CSFp may decrease further while SSp remains constant.

Thus, venous sinus narrowing in PTCS generates significantly raised CSFp in contrast to healthy normal subjects [20, $22,24]$. There are other conditions in which SSp may not remain constant during CSF infusion as in infants and in the presence of an open fontanelle, myelomeningocoele, or Chiari malformation, and in individual cases $[1,5,27,28,31]$. On the other hand, cerebral venous thrombosis and narrowing of the cerebral venous sinuses secondary, for example, to an intrasinus meningioma are recognised causes of PTCS where $\mathrm{SSp}$ is elevated but unchanged during CSF infusion.

\section{Coupling of the two pressures}

After the start of infusion, even though there is a direct coupling between changes in CSFp and SSp, the two pressures appeared to diverge compared with baseline (as shown in Fig. 1). This divergence between mean CSFp and SSp may reflect that, at the beginning of the CSF infusion, all the infused fluid is initially accommodated within the intracranial compliant space. As CSFp increases towards its plateau, the infused CSF is absorbed into the sagittal and transverse sinuses $[2,3,6]$.

\section{Implications of coupling between CSFp and SSp for the calculation of CSF outflow resistance}

Davson's equation refers to the steady state and assumes that $\mathrm{SSp}$ is independent of CSFp:

$\mathrm{CSFp}=$ Rout $\times \mathrm{I}_{\mathrm{f}}+\mathrm{SSp}$ 


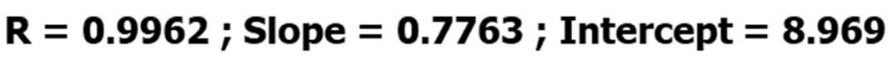

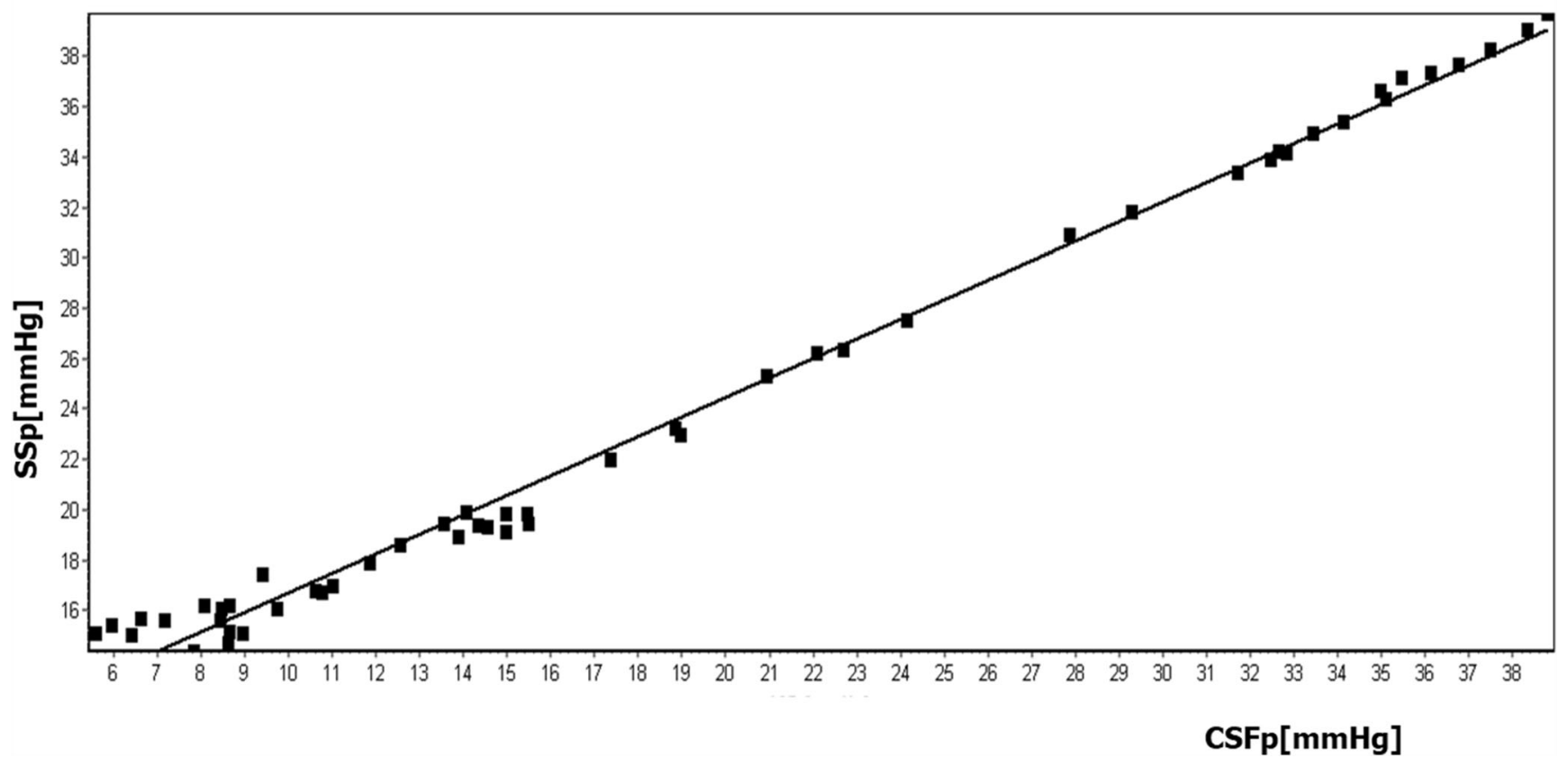

Fig. 4 Example of the linear regression analysis between CSFp (ICP in the figure) and SSp (venous in the figure) for 1 out of the 9 studied patients. The slope and the intercept of the regression for each patient were averaged and were used to express $\mathrm{SSp}$ as $\mathrm{SSp}=a \times \mathrm{CSFp}+b, a=$ slope and $b=$ intercept. SSp, expressed this way, can be used in Davson's

where Rout is the resistance to CSF outflow and $I_{\mathrm{f}}$ is the CSF formation rate [6].

In hydrocephalus, when SSp is not coupled to CSFp, a constant-rate infusion study enables the calculation of Rout as:

Rout $=\left(\operatorname{CSPp}_{\text {plateau }}-\mathrm{CSFp}_{\text {baseline }}\right) /$ Infusion rate

In the ten patients with PTCS in this study, the Rout calculated without correction for changes in $\mathrm{SSp}$ is $16.1 \pm 2.1$ $\mathrm{mmHg} /(\mathrm{ml} / \mathrm{min})$. Using the measured values of SSp, Rout is calculated as $5.2 \pm 1.4 \mathrm{mmHg} /(\mathrm{ml} / \mathrm{min})(p<0.001)$, approximately $67 \%$ lower.

One approach is to modify Davson's equation by expressing SSp as a function of CSFp (Fig. 4: SSp $=\mathrm{a} \times \mathrm{CSFp}+\mathrm{b}$ ). Therefore, Davson's equation may be rewritten for PTCS as:

$\mathrm{CSFp}=$ Rout $\times \mathrm{I}_{\mathrm{f}}+\mathrm{a} \times \mathrm{CSFp}+\mathrm{b}$

and subsequently,

$\mathrm{CSFp}=\left(\right.$ Rout $\left.\times \mathrm{I}_{\mathrm{f}}+\mathrm{b}\right) /(1-\mathrm{a})$

The average product of Rout $\times \mathrm{I}_{\mathrm{f}}$ is $3 \mathrm{mmHg}$ [8], $1.5 \mathrm{in} \mathrm{IIH}$ with the corrected Rout (see above); therefore, the average CSFp $=(1.5+6.3) / 0.3=26 \mathrm{mmHg}$. equation to simplify the calculations of its parameters in IIH patients. Notice that below CSFp $10 \mathrm{mmHg}$, SSp stopped to decrease further, while CSFp was easily drawn down by further drainage (JVP was $8 \mathrm{mmHg}$ in this patient, CVP was $\sim 11 \mathrm{mmHg}$ )

\section{Pathophysiological interpretation of Davson's equation in PTCS}

Finally, as derived from the simplified Davson's equation for IIH, CSFp is increased and is estimated to be around $26 \mathrm{mmHg}$. This estimation is almost identical to the mean baseline CSFp in our patients, which is calculated as $27 \mathrm{mmHg}$. The derived formula explains why in IIH with CSFp-SSp coupling, the baseline intracranial pressure is elevated. Moreover, correcting Rout as derived by Davson's equation, gives a more realistic estimation of a Rout on average $<7 \mathrm{mmHg} \times \mathrm{min} / \mathrm{ml}$. We have observed in most of our classic IIH patients, that CSFp at plateau is generally not much higher than baseline, resembling the normal CSF circulation, as opposed to hydrocephalus patients. In few exceptional cases, where a higher than expected CSF plateau is observed, knowledge of the SSp could provide valuable information about the differential diagnosis.

Malm and colleagues [23], using a constant pressure infusion technique, demonstrated that there may be two groups of PTCS patients - one group with genuinely reduced conductance (increased Rout) and a second group with increased SSp as the cause of their impaired CSF absorption. They also showed that changes in CSF conductance changed with time after onset of PTCS. 
However, these interpretations do not take into account the spatially distributed nature of CSF absorption. In most situations, CSF absorption is probably mainly intracranial and there is only a small gradient of pressure between the sagittal sinus and jugular foramen so that it is reasonable to use one single value for SSp. In contrast, in PTCS, there may be two CSF absorption pressure gradients - above and below an area of sinus narrowing. In other words, one Davson's equation is required to describe CSF absorption upstream of the stenosis and another Davson's equation for downstream absorption. Davson's equation assumes that all the infused CSF is drained through channel(s) that may be described by a single parameter. If CSF absorption is split between upstream and downstream channels, Davson's equation cannot be used as the relative proportion of ' $I_{\mathrm{f}}$ ' drained by the two systems is unknown. The transverse sinus pressure/JVP below the level of the stenosis is much lower than SSp above the stenosis. Lublinsky and colleagues have recently demonstrated the presence of arachnoid granulations in the transverse sinuses in both normal subjects and patients with IIH [21]. Interestingly, the total volume and interface contact area of intracranial arachnoid granulations is increased in IIH patients.

The situation may become even more complex if the stenosis is reversible with CSF removal and behaves as a Starling resistor [29]. If part of the transverse sinus is compressible, any rise in CSFp can decrease its lumen and increase the hydrodynamic resistance for sinus blood flow, increasing in the same way the SSp (if cerebral blood flow stays constant), which in turns increases CSFp. This mechanism works as a 'vicious circle' until CSFp and SSp reach an elevated state of equilibrium. This has been previously numerically simulated using an elegant mathematical model. The model forecasted that the system with collapsible transverse sinus (represented as a 'Starling Resistor') has two steady states: at low and at high CSFp.

\section{CSFp-SSp coupling in other intracranial pathologies}

An important question that merits systematic study is whether the phenomenon of direct coupling of CSFp to SSp is limited only to PTCS or may also play a role in some cases of acute intracranial hypertension seen during brain swelling (head injury, stroke, meningitis, etc.). A study in this direction from early work suggested that $60 \%$ of ICP should be attributed to vascular mechanisms, rather than CSF circulatory component $[18-20,22,32]$. In one post-TBI patient, we anecdotally studied with double SSp and CSFp measurement during an infusion study; SSp appeared to stay constant when CSFp elevated.
Finally, our statistically strong and significant findings could have important implications for PTCS patients, both in the adult and paediatric populations, and it would be worth designing future randomised trials aiming at treating PTCS patients by stopping the reported pathophysiological coupling of the two pressures.

\section{Limitations}

We did not collect information about arterial blood pressure during the infusion studies and therefore its role in the interaction between the CSF and arterial/venous blood flows for these PTCS patients.

Unfortunately, full analysis of the frequencies of the CSFp and $\mathrm{SSp} / \mathrm{JVP}$ is not possible using retrospective data because we did not have information about the frequency properties of the two pressure measurement systems used: in CSFp, a short and wide manometer line and a LP needle was used; however, in SSP, a long thin catheter and external transducer. This makes accurate spectral analysis on CSFp-SSp questionable.

\section{Conclusion}

CSFp and SSp are coupled in PTCS, both at baseline and during infusion, forming a positive feedback loop that may be interrupted by CSF drainage. The implications of the calculation of CSF outflow resistance are discussed.

Author contributions ADL drafted the manuscript, retrospectively collected all the data, and analysed them. MC and ZC assisted in the concept, practical implementation, and design of the procedures, as well as data analysis and interpretation, and contributed to writing the manuscript. DK critically reviewed the manuscript, contributed her knowledge and expertise in the topic related to the data analysis and interpretation, and contributed to the final version of the manuscript. JDP partially contributed to the conception and design of this study, orchestration of the practical performance of the two tests together, and final version of the manuscript. $\mathrm{NJH}$ was the one who conceived and requested the performance of these studies, after having previously laid the foundations and pioneering venous pressure studies and stenting in such patients. Statistical analysis was performed by ADL and cross-checked with MC to assure accuracy.

Funding information MC is supported by a grant of the Korea Health Technology R\&D Project through the Korea Health Industry Development Institute (KHIDI), funded by the Ministry of Health \& Welfare, Republic of Korea (grant no. HI17C1790) and previously by grant no. FCA 234/95 from the Technology Foresight Challenge Fund and by Program grant no. MRC G9439390 from the Medical Research Council. JDP was an NIHR Senior Investigator (2009-2014) and is an Honorary Director of the NIHR Brain Injury Healthcare Cooperative (2012-2017), now MedTech Cooperative (2018-2023).

Data availability Unfortunately, we do not possess appropriate ethics in order to share our anonymised data from patients, as all studies were performed on a clinical indication and at the time of consent there was no statement asking permission for sharing. 


\section{Compliance with ethical standards}

All tests were performed as part of routine clinical management. All patients consented to the use of their data recordings for research purposes. At the time of this study, such consent did not include permission for data sharing.

Conflicts of interest $\mathrm{MC}$ has a partial financial interest in licensing $\mathrm{ICM}+$ software, the tool used to perform and analyse ICP monitoring and infusion tests in Cambridge.

Ethical approval For this type of retrospective study, formal consent is not required.

Informed consent Informed consent was obtained from all individual participants included in the study.

Open Access This article is distributed under the terms of the Creative Commons Attribution 4.0 International License (http:// creativecommons.org/licenses/by/4.0/), which permits unrestricted use, distribution, and reproduction in any medium, provided you give appropriate credit to the original author(s) and the source, provide a link to the Creative Commons license, and indicate if changes were made.

\section{References}

1. Alexander JS, Prouty L, Tsunoda I, Ganta CV, Minagar A (2013) Venous endothelial injury in central nervous system diseases. BMC Med 11(1):219

2. Czosnyka M, Whitehouse H, Smielewski P, Simac S, Pickard JD (1996) Testing of cerebrospinal compensatory reserve in shunted and non-shunted patients: a guide to interpretation based on an observational study. J Neurol Neurosurg Psychiatry 60(5):549-558

3. Czosnyka M, Czosnyka ZH, Momjian S, Pickard JD (2004) Cerebrospinal fluid dynamics. Physiol Meas 25(5):R51-R76

4. Czosnyka Z, Czosnyka M, Owler B, Momjian S, Kasprowicz M, Schmidt EA, Smielewski P, Pickard JD (2005) Clinical testing of CSF circulation in hydrocephalus. Acta Neurochir Suppl 95:247251

5. Dandy WE (1937) Intracranial pressure withoun brain tumor: diagnosis and treatment. Ann Surg 106(4):492-513

6. Davson H, Domer FR, Hollingsworth JR (1973) The mechanism of drainage of the cerebrospinal fluid. Brain 96(2):329-336

7. Ekstedt J (1977) CSF hydrodynamic studies in man. 1. Method of constant pressure CSF infusion. J Neurol Neurosurg Psychiatry 40(2):105-119

8. Ekstedt J (1978) CSF hydrodynamic studies in man 2 Normal hydrodynamic variables related to CSF pressure and flow. J Neurol Psychiatry 41:345-353

9. Friedman D, Liu GTDK (2013) Revised diagnostic criteria for the pseudotumor cerebri syndrome in adults and children. Neurology 81(13):1159-1165

10. Friedman DI, Liu GT, Digre KB (2013) Revised diagnostic criteria for the pseudotumor cerebri syndrome in adults and children. Neurology 81(13):1159 LP-1151165

11. Hayhurst C, Rowlands A, Rowe F (2009) Idiopathic intracranial hypertension. In: Cerebrospinal Fluid Disord. CRC Press, Boca Raton, pp 337-379
12. Higgins JN, Pickard J (2004) Lateral sinus stenoses in idiopathic intracranial hypertension resolving after CSF diversion. Neurology 62:1907-1908

13. Higgins JNP, Owler BK, Cousins C, Pickard JD (2002) Venous sinus stenting for refractory benign intracranial hypertension. Lancet 359(9302):228-230

14. Higgins N, Pickard J, Lever A (2013) Lumbar puncture, chronic fatigue syndrome and idiopathic intracranial hypertension: a crosssectional study. JRSM Short Rep 4(12):204253331350792

15. Janny P, Chazal J, Colnet G, Irthum B, Georget AM (1981) Benign intracranial hypertension and disorders of CSF absorption. Surg Neurol 15(3):168-174

16. Johnston I (1973) REDUCED C.S.F. ABSORPTION SYNDROME. Reappraisal of benign intracranial hypertension and related conditions. Lancet 302(7826):418-421

17. Johnston I, Owler B, Pickard J (2007) The pseudotumor cerebri syndrome: pseudotumor cerebri, idiopathic intracranial hypertension, benign intracranial hypertension and related conditions. Cambridge University Press, Cambridge. https://doi.org/10.1017/ CBO9780511544996

18. Karahalios DG, Rekate HL, Khayata MH, Apostolides PJ (1996) Elevated intracranial venous pressure as a universal mechanism in pseudotumor cerebri of varying etiologies. Neurol 46(1):198-202

19. King JO, Mitchell PJ, Thomson KR, Tress BM (1995) Cerebral venography and manometry in idiopathic intracranial hypertension. Neurol 45(12):2224-2228

20. Kristensen B, Malm J, Markgren P, Ekstedt J (1992) CSF hydrodynamics in superior sagittal sinus thrombosis. J Neurol Neurosurg Psychiatry 55(4):287-293

21. Lublinsky S, Kesler A, Friedman A, Horev A, Shelef I (2018) Quantifying response to intracranial pressure normalization in idiopathic intracranial hypertension via dynamic neuroimaging. J Magn Reson Imaging 47(4):913-927

22. Lueck C, McIlwaine G (2002) Interventions for idiopathic intracranial hypertension. Cochrane Database Syst Rev. https://doi.org/10. 1002/14651858.CD003434

23. Malm J, Kristensen B, Markgren P, Ekstedt J (1992) CSF hydrodynamics in idiopathic intracranial hypertension: a long-term study. Neurology 42(4):851-858

24. Martins AN, Kobrine AI, Larsen DF (2009) Pressure in the sagittal sinus during intracranial hypertension in man. J Neurosurg 40(5): 603-608

25. Owler BK, Parker G, Halmagyi GM, Dunne VG, Grinnell V, McDowell D, Besser M (2003) Pseudotumor cerebri syndrome: venous sinus obstruction and its treatment with stent placement. J Neurosurg 98(5):1045-1055

26. Owler BK, Parker G, Halmagyi GM, Johnston IH, Besser M, Pickard JD, Higgins J (2005) Cranial venous outflow obstruction and psudotumor cerebri syndrome. Adv Tech Stand Neurosurg 30: 107-174

27. Pickard J (1988) Physiology and pathophysiology of the cerebrospinal fluid. J Neurol Neurosurg Psychiatry 51(3):469-470

28. Pickard JD, Czosnyka Z, Czosnyka M, Owler B, Higgins JN (2009) In: Steiger H-J (ed) Coupling of sagittal sinus pressure and cerebrospinal fluid pressure in idiopathic intracranial hypertension - a preliminary report BT - Acta Neurochirurgica Supplements. Springer Vienna, Vienna, pp 283-285

29. Piechnik SK, Czosnyka M, Richards H, Whitfield P, Pickard J (2001) Cerebral venous blood outflow: a theoretical model based on laboratory simulation. Neurosurgery 49(5):1214-1223

30. Smielewski P, Lavinio A, Timofeev I, Radolovich D, Perkes I, Pickard JD, Czosnyka M (2008) ICM+: a flexible platform for 
investigations of cerebrospinal dynamics in clinical practice. Acta Neurochir Suppl 102:145-151

31. Wakerley B, Tan M, Ting E (2015) Idiopathic intracranial hypertension. Cephalalgia 35(3):248-261

32. Yilmaz S, Serdaroglu G, Unver H, Akcay A, Gokben S, Tekgul H (2011) Recurrent pseudotumor cerebri in childhood: a case of neuro-behçet disease complicated with thrombotic risk factors. J Child Neurol 26(7):881-884

\section{Comments}

In this study, the authors evaluate ten adult patients with pseudotumour cerebri syndrome (PTCS) with CSF infusion studies, and with parallel direct retrograde cerebral venography. CSF pressure and sagittal sinus pressure were recorded simultaneously at baseline and during infusion; they were also recorded during drainage of CSF after completion of the infusion. The authors conclude that in patients with PTCS, CSF, and sagittal sinus pressure are coupled, with respect to both their static and dynamic components, during both baseline and infusion. They argue that elevated CSF pressure in PTCS may compress the venous sinuses, elevating CSF pressure further; this positive feedback loop is only interrupted by CSF drainage. They also argue that in PTCS, calculation of Rout must incorporate this coupling of CSF pressure with venous sinus pressure and demonstrate that only when this coupling is taken into consideration, do the derived values of Rout and CSF pressure mirror what is actually observed.

This is an important study, written by a group of researchers and clinicians who are well known for their expertise in, and previous scholarly contributions to, CSF dynamics and understanding of venous involvement in PTCS. It elegantly reviews the effects of PTCS on CSF dynamics, although stops short of discussing whether CSF infusion studies could be of any clinical value in the assessment of patients with PTCS. The review of Rout as applied to this condition is clear and succinct.

Kristian Aquilina

London, UK

Publisher's note Springer Nature remains neutral with regard to jurisdictional claims in published maps and institutional affiliations. 